\title{
Component Scheduling for Chip Shooter Machines: A Hybrid Genetic Algorithm Approach
}

\author{
William HO and Ping JI* \\ Department of Industrial and Systems Engineering \\ The Hong Kong Polytechnic University \\ Hung Hom, Kowloon, Hong Kong \\ FAX: (852)23625267, E-MAIL: MFPJI@POLYU.EDU.HK
}

\begin{abstract}
A chip shooter machine for electronic component assembly has a movable feeder carrier, a movable X-Y table carrying a Printed Circuit Board (PCB), and a rotary turret with multiple assembly heads. This paper presents a hybrid genetic algorithm to optimize the sequence of component placements and the arrangement of component types to feeders simultaneously for a chip shooter machine, that is, the component scheduling problem. The objective of the problem is to minimize the total assembly time. The genetic algorithm developed in the paper hybridizes different search heuristics including the nearest neighbor heuristic, the 2-opt heuristic, and an iterated swap procedure, which is a new improved heuristic. Compared with the results obtained by other researchers, the performance of the hybrid genetic algorithm is superior in terms of the assembly time.
\end{abstract}

\section{Scope and purpose}

When assembling the surface mount components on a PCB, it is necessary to obtain the optimal sequence of component placements and the best arrangement of component types to feeders simultaneously in order to minimize the total assembly time. Since it is very difficult to 
obtain the optimality, a genetic algorithm hybridized with several search heuristics is developed. The type of machines being studied is the chip shooter machine. This paper compares the algorithm with a simple genetic algorithm. It shows that the performance of the algorithm is superior to that of the simple genetic algorithm in terms of the total assembly time.

Keywords: Genetic algorithms; Heuristics; Chip shooter machines; Printed circuit board manufacturing; Component scheduling.

*Author to whom all correspondence should be addressed. 


\section{Introduction}

Printed circuit boards (PCBs) are extensively used in a variety of products today. These products include, for instance, computers, telephones, robots, and electronic instruments. In order to survive in today's highly competitive electronic industry, a company must be able to adapt to its customers' changing needs such as smaller product size but with greater function and reliability. These needs force the Surface Mount Technology (SMT) to replace the pin-through-hole (PTH) technology. Surface mount components are more attractive because they allow higher board densities and faster transmission speeds. Moreover, the needs drive the assembly technologies of electronic components and printed circuit boards from manual operations to completely automated ones, including the numerically controlled machines for the placement of SMT components on printed circuit boards. The expense of the automated assembly equipment motivates the PCB manufacturer to optimize assembly processes in order to either minimize the total production cost or maximize the profit.

Since the placement operation is the most time consuming one among the assembly processes and has the greatest potential on production cost reduction, many researchers have investigated the problems related to the placement operation. The placement operation consists of the following three basic steps or sub-problems [1]:

(i) allocation of components to machines,

(ii) arrangement of component types to feeders at each machine,

(iii) pick and placement sequencing.

In this paper, the second and the third sub-problems, in the SMT environment, are studied. For the PTH technology, the sequence of the insertion operation in the auto-insertion machine can be simply formulated as the traveling salesman problem [2], and it is not necessary to consider the feeder arrangement problem. However, in the SMT environment, the efficiency of component scheduling is also dependent on the feeder location to hold which types of components besides the pick and placement sequence. If the arrangement of components to 
feeders is not made carefully, even if the pick and placement sequencing is optimally solved, it can result in an extremely poor performance [3]. So, certainly, the problem of component placement sequencing as well as the feeder arrangement should be simultaneously solved.

\section{Literature review}

The problem of component placement sequencing and the feeder arrangement for a turret machine with a movable feeder and a movable board table, that is, a chip shooter machine, has been studying for nearly a decade. Bard et al. [4] used an iterative two-step approach to determine the component placement sequence, feeder arrangement, and retrieval plan for a chip shooter machine. Initially, a placement sequence was generated with a weighed nearest neighbor Traveling Salesman Problem (TSP) heuristic, whereas the remaining two problems were then formulated as a quadratic integer program and solved with a Lagrangian relaxation scheme. In the final step, the previous feeder arrangement was used to update the placement sequence, and the entire process was repeated. Souza and $\mathrm{Wu}$ [5] studied the component sequencing problem only for a chip shooter machine. A knowledge-based Component Placement System (CPS) incorporated with TSP algorithms was developed to solve the problem. They pointed out that the CPS is more practical and effective when compared with the machine proprietary algorithm. Moyer and Gupta [6] studied the feeder arrangement problem only for a chip shooter machine based on the assumption that the placement sequence of the components was predetermined. The problem was formulated as the Quadratic Assignment Problem (QAP). Two heuristic methods were proposed to solve the problem. Moyer and Gupta [7] proposed an asynchronous (acyclic) model for the component sequencing and feeder arrangement problem simultaneously, and developed a heuristic algorithm called the Acyclic Assembly Time (AAT) algorithm. In the acyclic model, if one of the operations such as the board is still moving to the proper position, the other mechanism is allowed to advance to the next position rather than to wait. Sohn and Park [8] formulated the component sequencing 
and feeder arrangement problem as a nonlinear integer programming model for a chip shooter machine while using the one-head case as an approximation to the multi-head case. A heuristic approach was developed to solve the problem. Yeo et al. [9] developed a rule-based frame system for PCB assembly to generate the component placement sequence and feeder arrangement for a chip shooter machine. The approach was based on the one-pitch incremental feeder heuristic and the nearest neighbor heuristic. Crama et al. [10] proposed a solution procedure based on a hierarchical decomposition of the planning problems for a chip shooter machine. They solved the feeder arrangement problem heuristically first, and then solved the problems of component placement sequence and component retrieval plan using constructive heuristics and local search methods. Dikos et al. [11] formulated the feeder arrangement problem for a chip shooter machine as the QAP, and made an assumption that an optimal component placement sequence was first specified. They employed genetic algorithms to find a near optimal feeder arrangement. Moyer and Gupta [12] agreed that the component sequencing problem for a chip shooter machine could be formulated as the TSP provided that the locations of the components on the board were assigned prior to the determination of the placement sequence. The Board Sequencing Heuristic (BSH) was developed to solve the problem. The idea of the BSH was to rearrange the placement order by swapping a pair of components in the current tour in order to obtain a better solution. Klomp et al. [13] treated the problem of determining an optimal feeder arrangement for a line of chip shooter machines as finding a shortest Hamiltonian path. An insertion heuristic and a local search heuristic were employed to solve the problem. Ellis et al. [14] developed a heuristic approach to determine the component placement sequence and feeder arrangement for a chip shooter machine. The nearest neighbor heuristic and the QAP greedy heuristic were used to generate the initial placement sequence and the initial feeder arrangement, respectively. The 2-opt local search heuristic was used to improve the initial solution.

In this paper, a hybrid genetic algorithm, which incorporates with three heuristics, namely, 
the nearest neighbor heuristic (NNH), the 2-opt local search heuristic, and a newly developed heuristic called the iterated swap procedure (ISP), is implemented to solve the component scheduling problem for a chip shooter machine, which combines the component sequencing problem and the feeder arrangement problem. This paper is organized as follows. Section 3 provides an overview of two types of placement machines. Section 4 describes the hybrid GA for the component scheduling problem. Section 5 discusses the result from the hybrid GA, and compares it with the result obtained by Leu et al. [15]. Section 6 concludes the paper.

\section{PCB assembly machines}

In the SMT environment, there are mainly two types of placement machines. Each type of machines possesses its own characteristics as well as operations. The first type of machines is called the pick and place (PAP) machine. In this type of machines, the components are stored in multiple stationary feeders. The placement head travels to pick up a component from a feeder at a time, and then place it on the stationary board. Although the speed of the PAP machine is slower than that of the chip shooter machine, the PAP machine is able to achieve higher accuracy, and suitable to operate with large components such as Integrated Circuit (IC). Jeevan et al. [16] proposed a genetic algorithm for PCB component placement for a PAP machine.

The second type of machines, to be studied in this paper, is called the chip shooter machine, as illustrated in Fig. 1. The major advantage of the chip shooter machine is its high speed and it is preferable to operate with small components such as chip resistors. The machine has a movable feeder carrier, a movable $\mathrm{X}-\mathrm{Y}$ table carrying a PCB, and a rotary turret with multiple assembly heads (usually 10 or 12). Each assembly head has several (normally 5) nozzles with different sizes. A large nozzle is used to pick up and place large components. The operation sequence of the chip shooter machine is that: As the first board of a batch enters the machine, the first nozzle of the turret picks up a component from a feeder. Then the turret 
indexes one step and the next nozzle picks up the second component. After that the turret indexes again to pick up the next component, and so on. In the same moment, the PCB is moved to the placement location waiting for the first component to be placed on the board. After the fifth component is picked up if the turret has 10 heads, the first component is placed on the board. These operations continue such as the turret indexes one step, the feeder carrier moves to the location containing the next pick-up component, and the $\mathrm{X}-\mathrm{Y}$ table moves to the next placement location. In the assembly of the last four components, there is no need to pick up components for the board being assembled. However, the nozzles of the turret pick up the first four components for the next board to be assembled.

For the first few components assembled in a batch of PCBs, there are only pick-up movements and no placement movement. For the last few components of the same batch, there are only placement movements and no pick-up movement. Therefore, if the quantity of PCB's in a batch is very large, these boundary effects can be neglected [15].

Since the chip shooter machine has three movable mechanisms, three different traveling times in the assembly of a component have to be considered simultaneously. They include the traveling time of PCB or the X-Y table, the traveling time of feeder carrier, and the shifting time of the tool heads (the indexing time of the turret), and the longest one among the three is the time for one component assembly, which is called the dominating time. The summation of the dominating times for all board components is the total assembly time needed for a PCB. Obviously, the objective of the component scheduling problem for this machine is to minimize the total assembly time such that the highest productivity of the machine can be achieved.

\section{Hybrid genetic algorithm}

In the recent years, Genetic Algorithms (GA), invented by John Holland in the 1960s, have been successfully applied to a wide variety of optimization problems such as the QAP, the minimum spanning tree problem, and the TSP [17-21]. The success is critical due to GA's 
simplicity, easy operation, and great flexibility. Genetic Algorithms are stochastic optimization techniques [17-21]. A GA starts with an initial set of random solutions, called population. Each solution in the population is called a chromosome, which represents a point in the search space. The genetic search progress is obtained by two essential genetic operations: exploitation (or intensification) and the exploration (or diversification). Generally, the crossover operator exploits a better solution while the mutation operator explores a wider search space.

The chromosomes evolve through successive iterations, called generations. During each generation, the chromosomes are evaluated by using some measures of fitness. The fitter the chromosomes, the higher the probabilities of being selected to perform a genetic operation (either crossover or mutation). In the crossover phase, the GA attempts to exchange portions of two parents (two chromosomes in the population) to generate an offspring. The crossover operation speeds up the process to reach better solutions. In the mutation phase, the mutation operation maintains the diversity in the population to avoid being trapped in a local optimum. A new generation is formed by selecting some parents and some offspring according to their fitness values, and by rejecting others to keep the population size constant. After the predetermined number of generations is performed, the algorithm converges to the best chromosome, which hopefully represents the optimal solution or may be a sub-optimal solution to the problem.

As mentioned before, a GA has a great level of flexibility. It can be hybridized with other heuristics in order to improve the solution further. However, it was found that none of the researchers have studied the component scheduling problem for the chip shooter machine by using a hybrid GA (HGA). So, this paper proposes a HGA to solve the problem. Besides, the performance of the HGA is compared with the simple GA.

The idea of the HGA for the component scheduling problem is described as follows. First of all, a two-link structure of representing a chromosome is used in the HGA for the problem. The first link represents the sequence of the component placements while the second link 
represents the feeder arrangement. The HGA starts with an initial population in which the first links in the chromosomes are generated from the nearest neighbor heuristic while the second links are generated randomly. During this initialization step, each chromosome is improved as follows: a new improved heuristic, called the iterated swap procedure (ISP), is performed on the first link while the 2-opt local search heuristic is applied to the second link. Each chromosome is then measured by an evaluation function. The roulette wheel selection operation is adopted to select some chromosomes for the genetic operations including one crossover and two mutations. After an offspring is produced, the first link is improved by the ISP while the second link is improved by the 2-opt local search heuristic. The fitness of the offspring will be measured and may become a member of the population if it possesses a relatively good quality. These steps form one iteration, and then the roulette wheel selection is performed again to start the next iteration. The HGA will not stop unless the predetermined number of iterations is conducted. The detailed algorithm is discussed in the following sections.

\subsection{Encoding}

A chromosome is illustrated in Fig. 2 with the two-link representation, in which the number inside the bracket in link 1 represents the component type. The path representation is used to encode the solution to the component scheduling problem. The idea of the path representation is that the components are listed in the order in which they are placed. For example, suppose there are 5 components numbered as $1,2,3,4$, and 5 . If the sequence of placements starts with component 3 , then component 1 , component 4 , component 5 , and finally

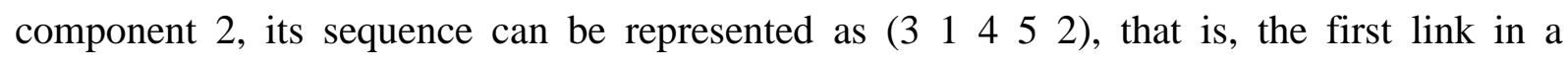
chromosome, as shown in Fig. 2. Furthermore, if the five components are from three component types, then component 3 of type 2 is placed first, whereas the component 2 of type 2 is placed last. On the other hand, component type 2 is located at feeder 1 . Therefore, feeder 1 is 
moved to the pick-up location, and a nozzle picks up the first component of type 2 in feeder 1 , and the turret rotates one step. After that, feeder 2 is moved to the pick-up location, and the second nozzle picks up a component of type 3 . The feeder carrier moves and the turret rotates again to pick up the third component of type 3 and the fourth component of type 1 . At this moment, the PCB moves so that component number 3 is located at the placement location. After the fifth component of type 2 is picked up, the first component is placed.

As a consequence, the sequence of component placements, $c$, will be (3 145 2), while the sequence of the feeders to be visited by the placement head, $f$, will be (1 2223 1).

\subsection{Evaluation}

For the component scheduling problem, the fitness function should be the total assembly time, which is the summation of all dominating times of components. It is because three mechanisms of the chip shooter machine move at different speeds, such as the traveling time of the PCB or the $\mathrm{X}-\mathrm{Y}$ table, the traveling time of the feeder carrier, and the indexing time of the turret. The longest one among the three is the dominating time needed in the assembly of the component, as discussed earlier. Let $T_{i}$ be the time needed for the placement of component $i$ and $\operatorname{eval}\left(X_{h}\right)$ be the total assembly time or the fitness function for chromosome $X_{h}$ in the component scheduling problem, then

$$
\begin{aligned}
& T_{i}=\max \left(t_{1}(c(i-1), c(i)), t_{2}(f(i+g), f(i+g+1)), t_{3}\right) \\
& \operatorname{eval}\left(X_{h}\right)=\sum_{i=1}^{n} T_{i}
\end{aligned}
$$

where

$t_{1}(a, b)$ is the traveling time of the $\mathrm{X}-\mathrm{Y}$ table from component $a$ to component $b$

$$
t_{1}(a, b)=\max \left(\frac{\left|x_{b}-x_{a}\right|}{V_{x}}, \frac{\left|y_{b}-y_{a}\right|}{V_{y}}\right) \text { for Chebyshev metric, and }
$$

$V_{x}$ is the speed of the $\mathrm{X}-\mathrm{Y}$ table in the $x$ direction, and

$V_{y}$ is the speed of the $\mathrm{X}-\mathrm{Y}$ table in the $y$ direction, and 
$t_{2}(u, v)$ is the traveling time of the feeder carrier from feeder $u$ to feeder $v$

$$
t_{2}(u, v)=\frac{\sqrt{\left(x_{v}-x_{u}\right)^{2}+\left(y_{v}-y_{u}\right)^{2}}}{V_{f}} \text {, and }
$$

$V_{f}$ is the speed of the feeder carrier, and

$t_{3}$ is the indexing time of the turret, and

$n$ is the number of components, and

$c(i)$ is the location in the board for the ith component, and

$f(i)$ is the feeder location for the ith component type, and

$g$ is the number of components in the gap between the pick-up component and the placement component in the turret, and normally, $g=5$ or 6 .

In the above expression, $c(i-1)=c(n)$ when $i=1$. When $f(l)$ has $l>n$, where $l=i+g$ or $i+g+1, f(l)$ is replaced by $f(l-n)$, which represents the initial $g$ components of the next board in the batch.

\subsection{Selection}

The roulette wheel approach [17] is adopted in order to choose some chromosomes to undergo genetic operations. The approach is based on an observation that a roulette wheel has a section allocated for each chromosome in the population, and the size of each section is proportional to the chromosome's fitness. The fitter the chromosome, the higher the probability of being selected. It is true that the roulette wheel selection mechanism chooses chromosomes probabilistically, instead of deterministically. For example, although one chromosome has the highest fitness, there is no guarantee it will be selected. The only thing that is certain is that on the average a chromosome will be chosen with the probability proportional to its fitness. Suppose the population size is psize, then the selection procedure is as follows:

Step 1: Calculate the total fitness of the population: 


$$
F=\sum_{h=1}^{p s i z e} \operatorname{eval}\left(X_{h}\right)
$$

Step 2: $\quad$ Calculate the selection probability $p_{h}$ for each chromosome $X_{h}$ :

$$
p_{h}=\frac{F-\operatorname{eval}\left(X_{h}\right)}{F \times(p s i z e-1)}, \quad h=1,2, \ldots, p \text { pize }
$$

Step 3: $\quad$ Calculate the cumulative probability $q_{h}$ for each chromosome $X_{h}$ :

$$
q_{h}=\sum_{j=1}^{h} p_{j}, \quad h=1,2, \ldots, \text { psize }
$$

Step 4: $\quad$ Generate a random number $r$ in the range $(0,1]$.

Step 5: $\quad$ If $q_{h-1}<r \leq q_{h}$, then chromosome $X_{h}$ is selected.

\subsection{Genetic operations}

The genetic operators used in the algorithm for the component scheduling problem are one crossover and two mutations, which are called the heuristic mutation and the inversion mutation, respectively. The two links in a chromosome are required to perform these genetic operations. The number of chromosomes selected to perform the crossover and mutation operations depends on the crossover rate and the mutation rate, respectively, which are predetermined by the GA user. Let crossno and mut denote the numbers of chromosomes selected to undergo the crossover and the mutation, respectively, then $\mathrm{crossno}=\operatorname{round}(\mathrm{cr}$ $\times p s i z e)$ and $m u t=\operatorname{round}(m r \times p s i z e)$, where $c r$ is the crossover rate, and $m r$ is the mutation rate. Since a pair of chromosomes is required to undergo the crossover operation, the number of pairs of chromosomes, denoted as cross, is an integer, so

$$
\text { cross }= \begin{cases}\frac{\text { crossno }}{2} & \text { if crossno is even } \\ \frac{\text { crossno }-1}{2} & \text { otherwise }\end{cases}
$$

\subsubsection{The modified order crossover operation}

As shown in Fig. 3, the crossover operator adopted in the HGA is a modified version of 
the classical order crossover operator, and two offspring will be generated at each time.

The procedure of the modified order crossover operation is listed as follows:

Step 1: Select a substring from the first parent randomly.

Step 2: Produce a proto-child by copying the substring into the corresponding positions in the proto-child.

Step 3: Find the gene right prior to the first gene of the substring from the second parent. If the gene is one of the genes in the substring, go to Step 4. Otherwise, place it in front of the substring in the proto-child.

Step 4: Find the gene right behind the last gene of the substring from the second parent. If the gene is one of the genes in the substring, go to Step 5. Otherwise, place it just after the substring in the proto-child.

Step 5: Delete those genes that are already in the proto-child from the second parent. The resulted genes, that is, the genes not in the proto-child yet, form a sequence.

Step 6: Place the genes into the unfilled positions of the proto-child from the left to the right according to the resulted sequence of genes in Step 5 to produce an offspring.

Step 7: Repeat Step1 to Step 6 to produce the second offspring by exchanging the two parents.

\subsubsection{The heuristic mutation operation}

A heuristic mutation is designed with the neighborhood technique in order to produce a better offspring. A set of chromosomes transformed from a parent by exchanging some genes is regarded as the neighborhood. Only the best one in the neighborhood is used as the offspring produced by the mutation. However, the purpose of the mutation operation is to promote diversity of the population. Therefore, it is necessary to make change to the original heuristic mutation for the component scheduling problem. The modification, as illustrated in Fig. 4, is that all neighbors generated are used as the offspring. The procedure of the heuristic mutation 
operation is listed as follows:

Step 1: Pick up 3 genes in a parent at random.

Step 2: Generate neighbors for all possible permutations of the selected genes, and all neighbors generated are regarded as the offspring.

Here, in Step 1, only 3 genes are selected since 2 genes has only one variation (one offspring) while more than 3 genes will generate too many offspring and it will take a very long time for computation.

\subsubsection{The inversion operation}

The inversion operator, as shown in Fig. 5, selects a substring from a parent and flips it to form an offspring. However, the inversion operator operates with one chromosome only, so it is very like the heuristic mutation and thus lacks interchange of the characteristics between chromosomes. So, the inversion operator is a mutation operation, which is used to increase the diversity of the population rather than to enhance the quality of the population.

\subsection{Improved heuristics}

Although the component scheduling problem is simple to describe verbally, it is very difficult to solve optimally when the number of components or the problem size is large. The improved heuristics are therefore helpful to tackle with this problem. In the HGA, three types of heuristics are adopted to improve the solution, including the nearest neighbor heuristic, the 2-opt local search heuristic, and the iterated swap procedure (ISP).

\subsubsection{The nearest neighbor heuristic}

The Nearest Neighbor Heuristic (NNH) is used to generate an initial solution for the first link only, which is the sequence of the component placements. The principle of the NNH is to start with the first component randomly, then to select the next component as close as possible 
to the previous one from those unselected components to form the placement sequence until all components are selected.

\subsubsection{The 2-opt local search heuristic}

Comparing with the total number of components in a PCB, the number of different component types is much fewer. Therefore, it is desirable to perform the 2-opt local search heuristic for the second link only, that is, the feeder arrangement. The principle of this heuristic is very straightforward. For one parent, all possible 2 swaps are examined to generate offspring and the best offspring will replace the parent if the offspring has a shorter assembly time than the parent.

\subsubsection{The iterated swap procedure}

The computational effort will be high if the 2-opt local search is performed for the first link, which is the sequence of component placements, because the number of components is quite large, normally several hundreds. As a consequence, a 'fast' improved heuristic is developed, which is called the iterated swap procedure (ISP). The ISP, as illustrated in Fig. 6, is performed for the first link of each initial solution generated by the $\mathrm{NNH}$ as well as each offspring generated by the three genetic operators. The procedure of the ISP is as follows:

Step 1: Select two genes randomly from the first link of a parent.

Step 2: Exchange the positions of the two genes to form an offspring.

Step 3: Swap the neighbors of the two genes to form four more offspring.

Step 4: Evaluate all offspring and find the best one.

Step 5: If the best offspring is better than the parent, replace the parent with the best offspring and go back to Step 1; Otherwise, Stop.

\subsection{The algorithm}


The procedure of the HGA for the component scheduling problem is listed as follows:

Step 1: Set the GA parameters, including the population size (psize), the number of iterations (itno), the crossover rate ( $\mathrm{cr}$ ), and the mutation rate $(\mathrm{mr})$.

Step 2: Generate psize initial chromosomes. For each chromosome, the first link is generated by the NNH in Section 4.5.1 while the second link is generated randomly.

Step 3: The 2-opt local search heuristic in Section 4.5.2 is performed on the second link for each initial chromosome.

Step 4: The ISP in Section 4.5.3 is performed on the first link for each initial chromosome.

Step 5: Evaluate the fitness value eval $\left(X_{h}\right)$ for all chromosomes in the population.

Step 6: Follow the selection procedure in Section 4.3 to select cross pairs of chromosomes to perform the modified order crossover operation in Section 4.4.1.

Step 7: Follow the selection procedure in Section 4.3 to select mut chromosomes to perform the heuristic mutation operation in Section 4.4.2.

Step 8: Follow the selection procedure in Section 4.3 to select mut chromosomes to perform the inversion operation in Section 4.4.3.

Step 9: The 2-opt local search heuristic in Section 4.5.2 is performed on the second link for each offspring generated in Steps 6, 7, and 8.

Step 10: The ISP in Section 4.5.3 is performed on the first link for each offspring generated in Steps 6, 7, and 8 .

Step 11: Compare all offspring with the chromosomes in the population by the fitness values $\operatorname{eval}\left(X_{h}\right)$. Retain the best psize chromosomes in the population.

Step 12: Find the best chromosome at each iteration. Repeat Step 6 to Step 12 until itno iterations are performed.

\section{Performance analysis}

The performance of the HGA was evaluated by use of the PCB example in [15]. The 
example has 50 components with 10 different component types.

\subsection{Effect of population size}

Population size is the number of chromosomes in the population. As mentioned earlier, a chromosome represents a point in the search space. Therefore, larger population size means more search points. The more the search points, the higher the chance of finding the optimal solution. However, larger population size needs longer computation times and more computer storage space is occupied. In order to identify the effect of the population size, the HGA program is run with two different population sizes. Fig. 7 and Fig. 8 show the performance of the HGA with the population sizes of 50 and 5, respectively. The parameters of the HGA for the problem are preset as: iteration number itno $=1,000$, crossover rate $c r=0.4$, and mutation rate $m r=0.2$.

It can be seen from the graphs that not only the HGA with the population size of 50 obtains a better chromosome in the initial population, but also it requires less number of iterations to obtain a better final solution. This may be due to the fact that more offspring are produced at each iteration. There are totally 80 offspring (20 from the modified order crossover operation, 50 from the heuristic mutation, and 10 from the inversion operation) produced at each iteration. However, there are only 8 offspring (2 from the modified order crossover operation, 5 from the heuristic mutation, and 1 from the inversion operation) produced at each iteration if the population size is 5 .

Although the HGA with the population size of 50 has higher convergence, the amount of time spent on a 700Mhz computer is ten times compared with the HGA with the population size of 5. The comparison between the two different-sized HGAs is summarized in Table 1.

\subsection{Comparison of results}

The parameters of the HGA for the problem are preset as: population size psize $=25$, 
iteration number itno $=1,000$, crossover rate $c r=0.4$, and mutation rate $m r=0.2$. Therefore, 5 pairs of chromosome are selected to perform the modified order crossover operation, whereas 5 chromosomes perform the heuristic mutation and the inversion operation. The total number of offspring produced per iteration will be 40 (10 from the modified order crossover operation, 25 from the heuristic mutation, and 5 from the inversion operation). Fig. 9 shows the best assembly time at each iteration, whereas the comparison between the HGA result and solution in [15] is shown in Table 2.

According to Table 2, it is found that the performance of the HGA is superior to that of the simple GA used by Leu et al. [15] in four aspects. Firstly, the best chromosome (30s) in the initial population obtained by the HGA is better than that of the simple GA (about 70s). Secondly, the HGA can obtain a better solution with a smaller population size, 25 only, while the other one was 100 . Thirdly, the HGA can obtain a better solution not only with a smaller population size, but also with fewer iterations, 323 vs. 1750. Finally and the most importantly, the HGA obtained a better solution than the previous method, 26s vs. $51.5 \mathrm{~s}$.

\section{Conclusions}

A hybrid genetic algorithm incorporated with three different heuristics was applied successfully to the component scheduling problem, which is a combined problem of component sequencing and feeder arrangement in PCB assembly with the objective of minimizing the total assembly time for a chip shooter machine. The three heuristics are the nearest neighbor heuristic, the 2-opt local search heuristic, and a newly developed improved heuristic called the iterated swap procedure. Furthermore, a two-link representation together with the modified order crossover, the heuristic mutation, and the inversion mutation were used to solve the problem. Finally, it was shown that the performance of the HGA is superior to that of the simple GA in terms of the total assembly time. 


\section{Acknowledgements}

The authors wish to acknowledge The Hong Kong Polytechnic University for the financial support of the project (No. G-W031). 


\section{References}

[1] Ball MO, Magazine MJ. Sequencing of insertions in printed circuit board assembly. Operations Research 1988;36(2):192-201.

[2] Chan D, Mercier D. IC insertion: an application of the travelling salesman problem. International Journal of Production Research 1989;27(10):1837-41.

[3] Altinkemer K, Kazaz B, Köksalan M, Moskowitz H. Optimization of printed circuit board manufacturing: integrated modeling and algorithms. European Journal of Operational Research 2000;124:409-21.

[4] Bard JF, Clayton RW, Feo TA. Machine setup and component placement in printed circuit board assembly. The International Journal of Flexible Manufacturing Systems 1994;6: 5-31.

[5] Souza RD, Wu LJ. Intelligent optimization of component onsertion in multi-head concurrent operation PCBA machines. Journal of Intelligent Manufacturing 1995;6: 235-43.

[6] Moyer LK, Gupta SM. SMT feeder slot assignment for predetermined component placement paths. Journal of Electronics Manufacturing 1996;6(3):173-92.

[7] Moyer LK, Gupta SM. Simultaneous component sequencing and feeder assignment for high speed chip shooter machines. Journal of Electronics Manufacturing 1996;6(4):271-305.

[8] Sohn J, Park S. Efficient operation of a surface mounting machine with a multihead turret. International Journal of Production Research 1996;34(4):1131-43.

[9] Yeo SH, Low CW, Yong KH. A rule-based frame system for concurrent assembly machines. International Journal of Advanced Manufacturing Technology 1996;12:370-6.

[10] Crama Y, Flippo OE, Klundert JVD, Spieksma FCR. The assembly of printed circuit boards: a case with multiple machines and multiple board types. European Journal of Operational Research 1997;98:457-72. 
[11] Dikos A, Nelson PC, Tirpak TM, Wang W. Optimization of high-mix printed circuit card assembly using genetic algorithms. Annals of Operational Research 1997;75:303-24.

[12] Moyer LK, Gupta SM. An efficient assembly sequencing heuristic for printed circuit board configurations. Journal of Electronics Manufacturing 1997;7(2):143-60.

[13] Klomp C, Klundert JVD, Spieksma FCR, Voogt S. The feeder rack assignment problem in PCB assembly: a case study. International Journal of Production Economics 2000;64:399-407.

[14] Ellis KP, Vittes FJ, Kobza JE. Optimizing the performance of a surface mount placement machine. IEEE Transactions on Electronics Packaging Manufacturing 2001;24(3):160-70.

[15] Leu MC, Wong H, Ji Z. Planning of component placement/insertion sequence and feeder setup in PCB assembly using genetic algorithms. Journal of Electronic Packaging $1993 ; 115: 424-32$.

[16] Jeevan K, Parthiban A, Seetharamu KN, Azid IA. Quadir, GA. Optimization of PCB Component Placement Using Genetic Algorithms, Journal of Electronics Manufacturing 2002;11(1):69-79.

[17] Goldberg DE. Genetic algorithms in search, optimization and machine learning. New York: Addison-Wesley, 1989.

[18] Davis L. Handbook of genetic algorithms. New York: Van Nostrand Reinhold, 1991.

[19] Chambers L. Practical handbook of genetic algorithms, Volume II. Boca Raton, Florida: CRC Press, 1995.

[20] Mitchell M. Introduction to genetic algorithms. London: MIT Press, 1996.

[21] Gen M, Cheng R. Genetic algorithms and engineering design. New York: Wiley, 1997. 
William Ho is a research student at the Department of Industrial and Systems Engineering, The Hong Kong Polytechnic University. His research area is the production planning of the printed circuit board assembly.

Ping $\mathbf{J i}$ is an Associate Professor at the Department of Industrial and Systems Engineering, The Hong Kong Polytechnic University. His research areas include Production Management, Operations Research, and CAD/CAM. 
Fig. 1. The schematic diagram of the chip shooter machine.

Fig. 2. The two-link representation for a chromosome.

Fig. 3. The modified order crossover operator.

Fig. 4. The heuristic mutation operator.

Fig. 5. The inversion operator.

Fig. 6. The iterated swap procedure.

Fig. 7. The performance of the HGA with 50 population sizes.

Fig. 8. The performance of the HGA with 5 population sizes.

Fig. 9. The best assembly time at each iteration. 


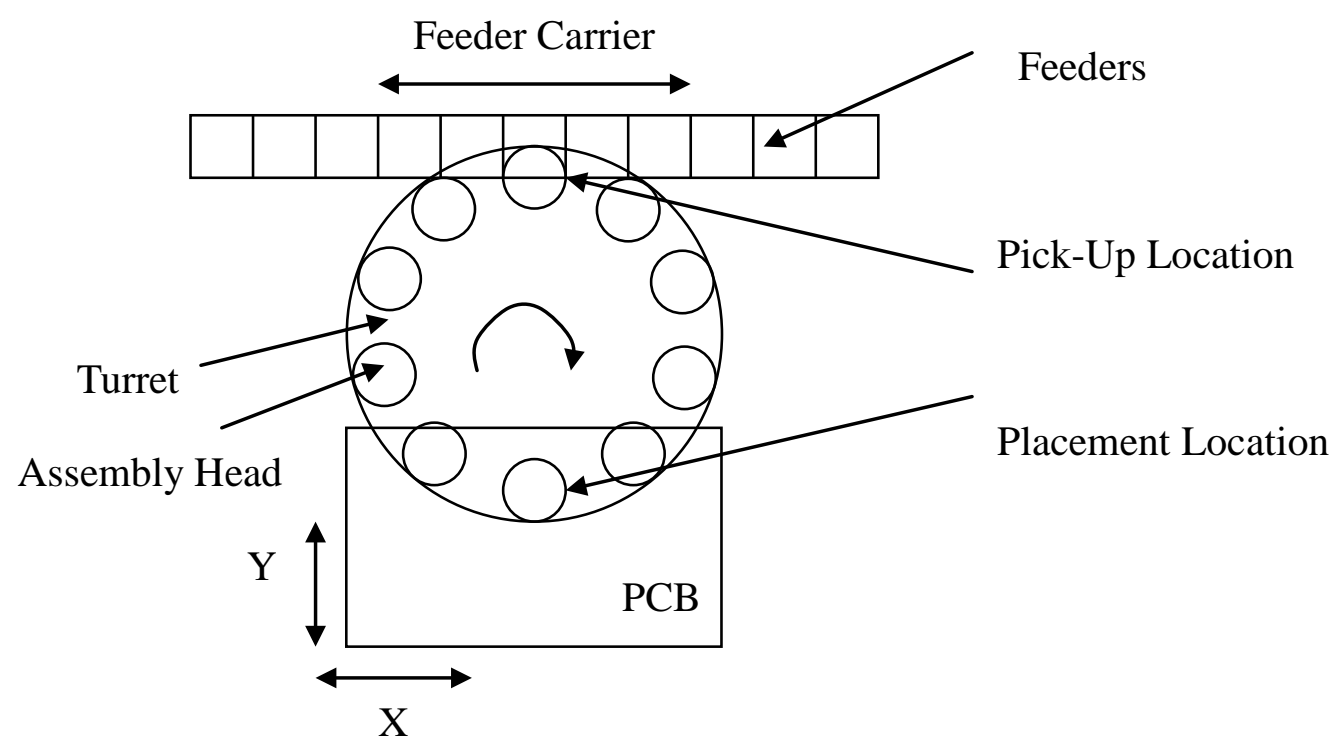

Fig. 1. The schematic diagram of the chip shooter machine. 


\begin{tabular}{|c|c|c|c|c|c|}
\hline Assembly Sequence & 1 & 2 & 3 & 4 & 5 \\
\hline Component Number (Component Type) & $3(2)$ & $1(3)$ & $4(3)$ & $5(1)$ & $2(2)$ \\
\hline & \multicolumn{5}{|c|}{ Link 1} \\
\hline Component Type & 2 & 3 & 1 & & \\
\hline Feeder Location & 1 & 2 & 3 & & \\
\hline
\end{tabular}

Fig. 2. The two-link representation for a chromosome. 
Selected substring

Parent 1:

Parent 2:

\begin{tabular}{llllll|lll}
1 & 2 & 3 & 4 & 5 & 6 & 7 & 8 & 9 \\
5 & 7 & 4 & 9 & 1 & 3 & 6 & 2 & 8
\end{tabular}

Offspring 1:

$\begin{array}{lllllllll}7 & 1 & 3 & 4 & 5 & 6 & 2 & 9 & 8\end{array}$

Parent 2:

Parent 1:

$\begin{array}{llllllllll}5 & 7 & 4 & 9 & 1 & 3 & 6 & 2 & 8 \\ 1 & 2 & 3 & 4 & 5 & 6 & 7 & 8 & 9 \\ 2 & 5 & \mathbf{4} & \mathbf{9} & \mathbf{1} & 3 & 6 & 7 & 8\end{array}$

Fig. 3. The modified order crossover operator. 
Select 3 genes at random

$\begin{array}{lllllllllllll}\text { Parent: } & 1 & 2 & 3 & 4 & 5 & 6 & 7 & 8 & 9 \\ & & & & & & & & & & & & \\ \text { Offspring 1: } & 1 & 2 & \mathbf{3} & 4 & 5 & \mathbf{8} & 7 & \mathbf{6} & 9 \\ \text { Offspring 2: } & 1 & 2 & \mathbf{6} & 4 & 5 & \mathbf{3} & 7 & \mathbf{8} & 9 \\ \text { Offspring 3: } & 1 & 2 & \mathbf{6} & 4 & 5 & \mathbf{8} & 7 & \mathbf{3} & 9 \\ \text { Offspring 4: } & 1 & 2 & \mathbf{8} & 4 & 5 & \mathbf{3} & 7 & \mathbf{6} & 9 \\ \text { Offspring 5: } & 1 & 2 & \mathbf{8} & 4 & 5 & \mathbf{6} & 7 & \mathbf{3} & 9\end{array}$

Fig. 4. The heuristic mutation operator. 
Selected substring

Parent:

$\begin{array}{lllllllll}1 & 2 & 3 & 4 & 5 & 6 & 7 & 8 & 9 \\ 1 & 2 & \mathbf{6} & \mathbf{5} & \mathbf{4} & \mathbf{3} & 7 & 8 & 9\end{array}$

Offspring:

Fig. 5. The inversion operator. 
Select 2 genes randomly

$\begin{array}{llllllllllll}\text { Parent: } & 1 & 2 & 3 & 4 & 5 & 6 & 7 & 8 & 9 \\ & & & & & & & & & & & \\ \text { Offspring 1: } & 1 & 2 & \mathbf{8} & 4 & 5 & 6 & 7 & \mathbf{3} & 9 \\ \text { Offspring 2: } & 1 & \mathbf{8} & \mathbf{2} & 4 & 5 & 6 & 7 & 3 & 9 \\ \text { Offspring 3: } & 1 & 2 & \mathbf{4} & \mathbf{8} & 5 & 6 & 7 & 3 & 9 \\ \text { Offspring 4: } & 1 & 2 & 8 & 4 & 5 & 6 & \mathbf{3} & \mathbf{7} & 9 \\ \text { Offspring 5: } & 1 & 2 & 8 & 4 & 5 & 6 & 7 & \mathbf{9} & \mathbf{3}\end{array}$

Fig. 6. The iterated swap procedure. 
Total assembly time

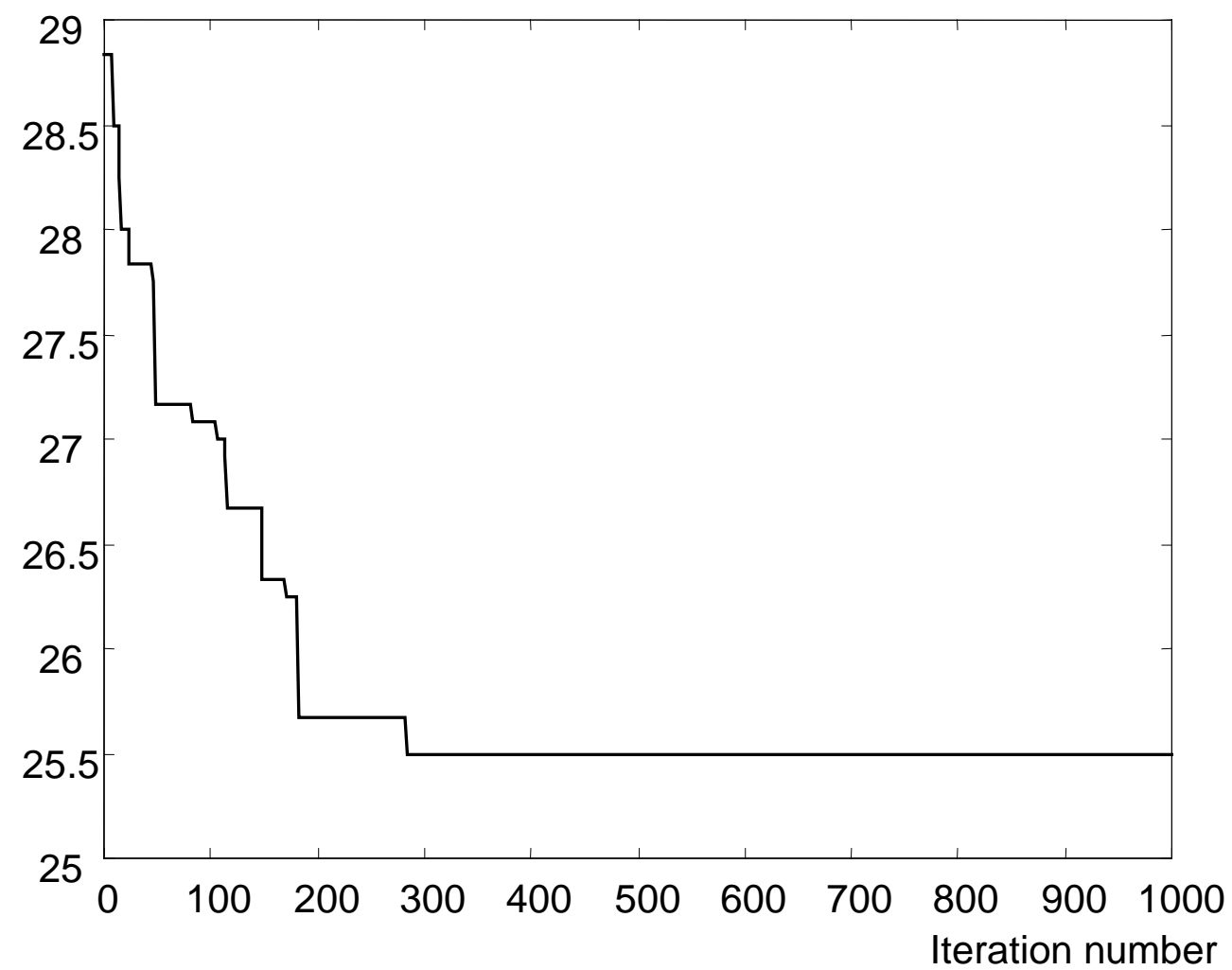

Fig. 7. The performance of the HGA with 50 population sizes. 
Total assembly time

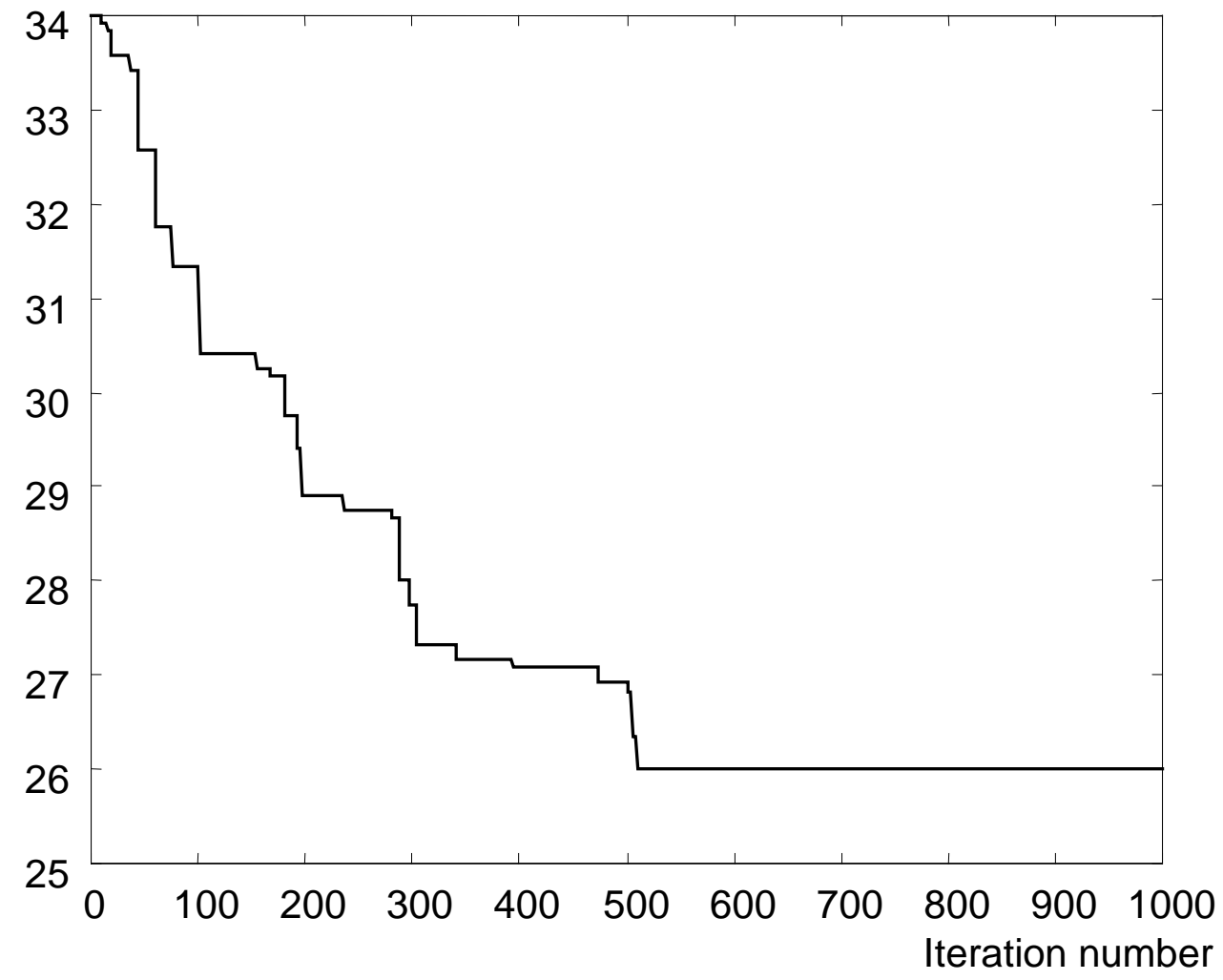

Fig. 8. The performance of the HGA with 5 population sizes. 
Total assembly time

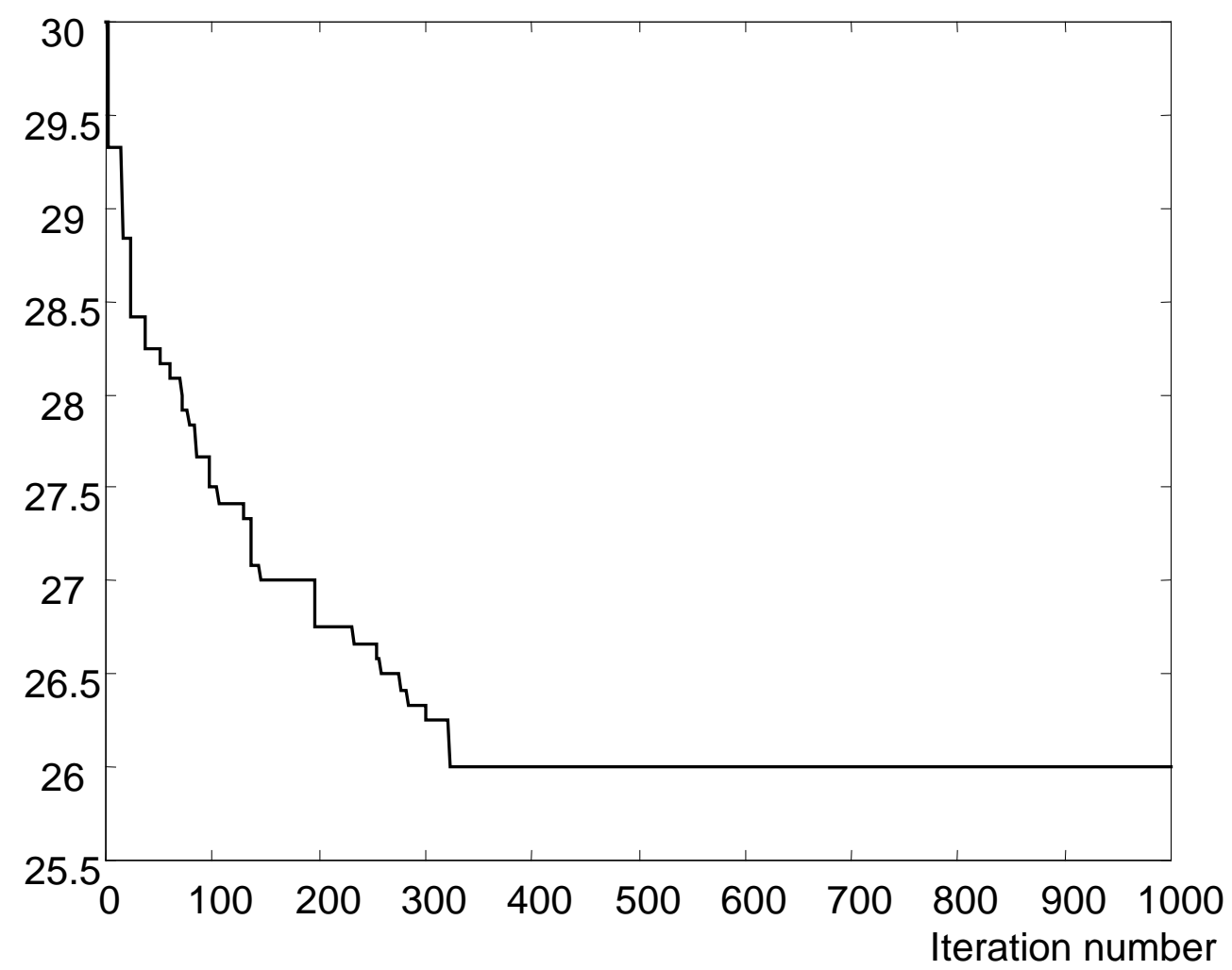

Fig. 9. The best assembly time at each iteration. 
Table 1

A comparison of two population sizes

\begin{tabular}{|rcc|}
\hline & psize $=50$ & psize $=5$ \\
Number of offspring produced at each iteration & 80 & 8 \\
Best one in the initial population (seconds) & 28.8333 & 34 \\
Final Best Solution (seconds) & 25.5 & 26 \\
Iteration number & 283 & 510 \\
Computation time (minutes) & 10 & 1 \\
\hline
\end{tabular}


Table 2

A comparison of the experimental results

\begin{tabular}{|rcc|}
\hline & Leu et al. [15] & HGA \\
Best one in the initial population (seconds) & 70 & 30 \\
Population size & 100 & 25 \\
Iteration number & about 1,750 & 323 \\
Final Best Solution (seconds) & about 51.5 & 26 \\
\hline
\end{tabular}




\section{University Library}

\section{- $\mathrm{M}$ IIN E R VA A gateway to Melbourne's research publications}

Minerva Access is the Institutional Repository of The University of Melbourne

Author/s:

Ho, W;Ji, P

Title:

Component scheduling for chip shooter machines: a hybrid genetic algorithm approach

Date:

2003-12-01

Citation:

Ho, W. \& Ji, P. (2003). Component scheduling for chip shooter machines: a hybrid genetic algorithm approach. COMPUTERS \& OPERATIONS RESEARCH, 30 (14), pp.2175-2189. https://doi.org/10.1016/S0305-0548(02)00129-6.

Persistent Link:

http://hdl.handle.net/11343/118698 